\title{
Bio-inspired fabrication of antireflection nanostructures by replicating fly eyes
}

\author{
Jingyun Huang ${ }^{1,2}$, Xudong Wang ${ }^{1}$ and Zhong Lin Wang ${ }^{1}$ \\ ${ }^{1}$ School of Materials Science and Engineering, Georgia Institute of Technology, Atlanta, \\ GA 30332-0245, USA \\ ${ }^{2}$ State Key Laboratory of Silicon Materials, Zhejiang University, Hangzhou 310027, \\ People's Republic of China \\ E-mail: zhong.wang@mse.gatech.edu
}

Received 30 August 2007, in final form 23 October 2007

Published 6 December 2007

Online at stacks.iop.org/Nano/19/025602

\begin{abstract}
In this study, we examined the fine structure of the compound eyes of a household fly and precisely replicated its entire structure using a low-temperature atomic layer deposition technique. The surface of the fly eye is found to be covered by highly packed protuberances, which potentially increases visual efficiency through increased photon capture for a given stimulus. The alumina replica was achieved by removing the fly compound eye template at high temperature, and the alumina coating was crystallized simultaneously. Besides the morphology, the unique antireflection property was also inherited by the alumina replica. By measuring the reflective spectra of the replica, we demonstrated that the alumina replica of a fly eye was an efficient antireflection structure of visible light at an incident angle up to $80^{\circ}$. Such a grating would be particularly useful on a cured corneal surface since it could increase the transmission of incident light through the cornea compared with a smooth surface.
\end{abstract}

(Some figures in this article are in colour only in the electronic version)

\section{Introduction}

Nature provides abundant selections of microstructures to nanostructures that can be used as templates for fabricating a wide range of optical structures [1-6]. Replication is a method of using biotemplates for achieving nanostructures made of more stable, harder, and high-temperature-tolerable inorganic materials that may have some designed functionalities for practical applications. Inorganic structures replicated from biological templates can combine the merits offered by both the material and biological structures for fabricating unique structures with much enhanced mechanical properties and finely tuned optical performance. Recently a photonic crystal structure has been successfully fabricated using butterfly wings as templates [7]. The replica of the butterfly wings also exhibited the same photonic crystal properties as their original wing template, which may be used as the building blocks for photonic integrated circuits with high reproducibility and lower fabrication cost compared to traditional lithography techniques.

In the insect world, the fly and butterfly have a very well-developed visual system with the capacity of seeing motion, color and pattern of objects in their environment due to their advanced compound eyes [8]. The compound eye is comprised of thousands of small eyes, each a so-called ommatidium. Each ommatidium consists of a light-diffracting facet lens, a crystalline cone, and photoreceptor cells with a wave-guiding rhabdom [9]. Compound eyes in nature present attractive physiological optics in high sensitivity and antireflection because of their final compound eye structure. In this study, we have precisely replicated the compound eye structures of a household fly with a wide field of vision of $150^{\circ}$ using atomic layer deposition (ALD). The reflectance spectra measurement demonstrated that the replica preserved the antireflection optical property of the original fly eyes. A reflection peak was discovered in the near-ultraviolet (UV) region due to the periodic grating structure. The reflectance spectra showed efficient antireflection property in the visible light and near-infrared (IR) range (400-1100 nm). An optical diffractive equation was used to explain it. This discovery showed great potential in applying the replicated fly compound eyes as the optical devices for a sensor or lens array. 


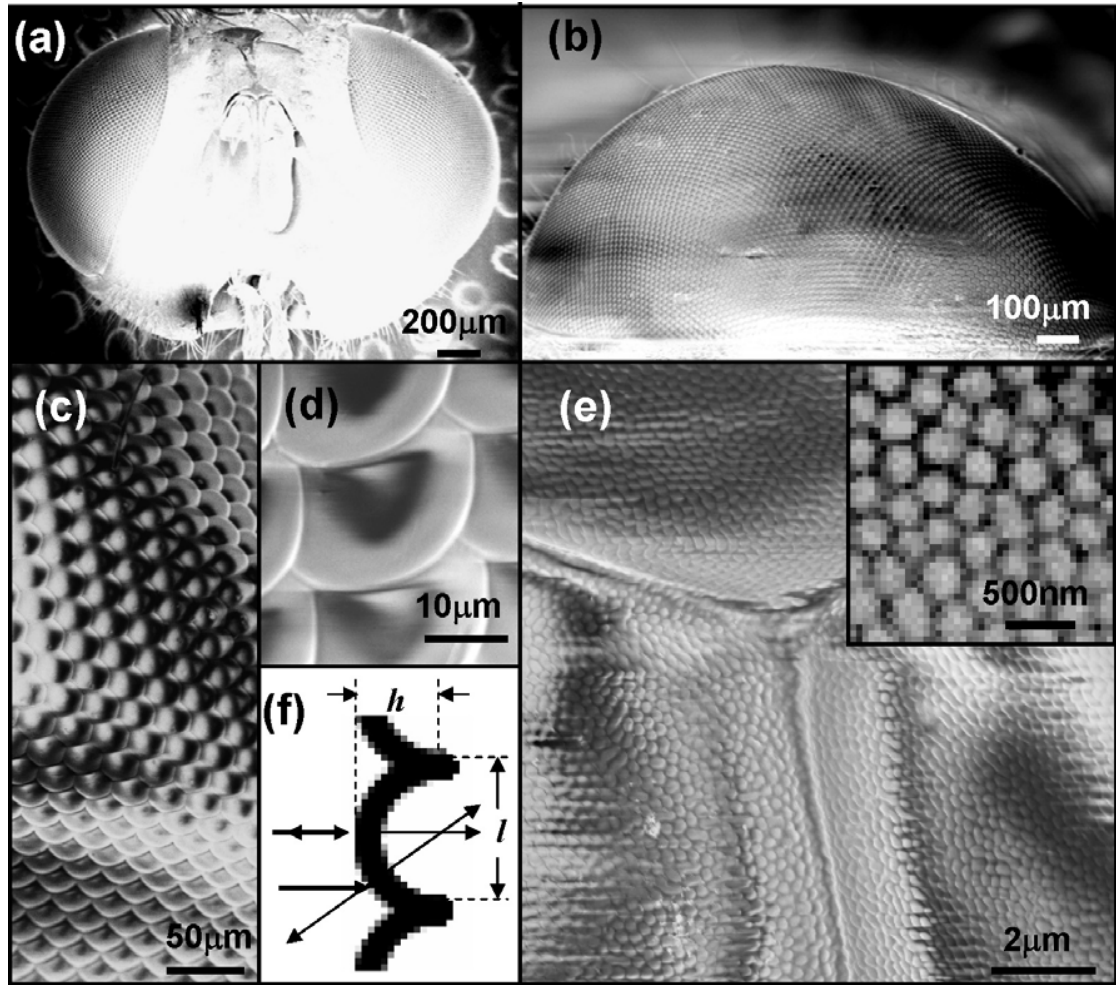

Figure 1. Structure of a household fly eye. (a) Low magnification SEM image of the fly's head. (b) Low magnification SEM image of the single fly frontal eye showing the compound eye structure. (c) Array of the ommatidia on the compound eye. (d) Enlarged image of the ommatidia structure. (e) High magnification SEM image of the ommatidia surface showing the protuberance nanostructures. Inset: enlarged SEM image of the protuberances on ommatidia surface. (f) Schematic of the protuberance's hemisphere nanostructure and the possible light transmission diagram.

\section{Experimental details}

The original fly compound eye sample was acquired from a household fly and fixed on an alumina substrate by carbon tape. The ALD deposition was performed in a Savannah 100 Atomic Layer Deposition system. $\mathrm{Al}\left(\mathrm{CH}_{3}\right)_{3}$ (TMA) and deionized water $(R \approx 18 \mathrm{M} \Omega$ ) were used as precursors for alumina growth. The deposition was performed at $80^{\circ} \mathrm{C}$ and under $\sim 0.6$ Torr chamber pressure. Ultra high purity $\mathrm{N}_{2}$ was used as carrying and purging gas with a flow rate of $20 \mathrm{sccm}$. The pulse duration was $0.03 \mathrm{~s}$ for each precursor and the purge time was $25 \mathrm{~s}$ between pulses. The average growth rate of alumina was $\sim 1 \AA$ per cycle. After deposition, the original fly eye template was removed by annealing the sample in a convention box furnace at $500^{\circ} \mathrm{C}$ for $15-20 \mathrm{~h}$.

The morphologies of fly compound eyes and their alumina replica were characterized by LEO 1530 field emission scanning electron microscopy (SEM) operated at $3 \mathrm{keV}$ and Hitachi HF2000 transmission electron microscopy (Hitachi 2000) operated at $200 \mathrm{keV}$. The chemical composition and crystallography were characterized by energy dispersive $\mathrm{x}$ ray (EDX) spectroscopy during the TEM analysis. The optical images were taken on a Nikon Eclipse L150 optical microscope with dark field function. The UV-visible reflection spectrum was measured in a Beckman DU640 spectrophotometer equipped with angle adjusting adaptor. The original fly eye sample for reflection measurements was left in a droplet of ethanol and seamlessly attached to a silicon substrate by capillary force during ethanol drying. After ALD deposition, the alumina replica was grown together with the silicon substrate so that no other intermediate interference was introduced to the reflection measurement. The referencing reflection was measured on the alumina thin film on the same silicon substrate, where it is not covered by the fly eye replica.

\section{Results and discussion}

A household fly's eye has thousands of integrated optical units called ommatidia spherically arranged along a curvilinear surface, and the ommatidia are arranged in a hexagonal pattern. The low magnification SEM image in figure 1(a) shows the head of a fly. Its compound eye is shown in figure 1(b). The size of a single fly eye is about $1.2 \mathrm{~mm}$ in diameter, with the field of vision about $150^{\circ}$. Such a wide field of vision exhibits a huge potential for medical, industrial, and defense applications. The ommatidium structure on the fly eye is shown in figure 1(c). They exhibit a highly ordered arrangement across the entire eye surface. As shown in figure $1(\mathrm{~d})$, a typical ommatidium is almost a hemisphere with a length of $\sim 21 \mu \mathrm{m}$ and a height of $\sim 9 \mu \mathrm{m}$, from which the angle of view (arc of the ommatidium) can be determined to be $141^{\circ}$. Under a higher magnification SEM image, it was found the surface of an ommatidium is not smooth; instead, it is covered by highly packed nanosized 


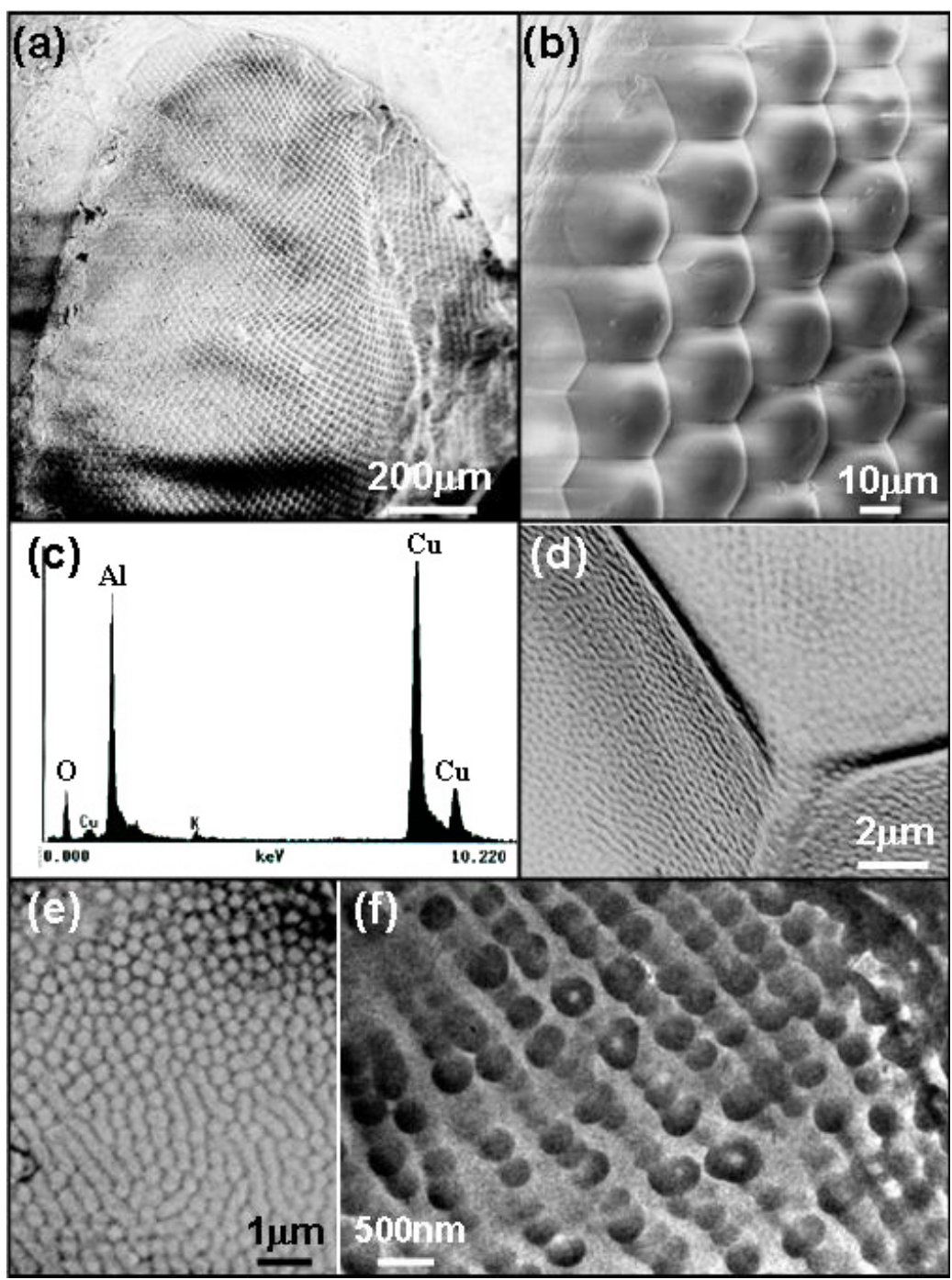

Figure 2. Structure of the alumina replica of the fly eyes. (a) SEM image of the alumina replica of a fly compound eye. (b) SEM image of the alumina replica showing that the array of ommatidia was perfectly preserved from the original fly eye. (c) EDS of the alumina replica. (d) High magnification SEM image of the alumina replicated ommatidium surface with protuberances. (e) Enlarged SEM image showing the replicated protuberance nanostructures. (f) TEM image of a piece of replicated ommatidium structure showing the linear arrangement of spherical the protuberances.

protuberant hemispheres, as shown in figure 1(e). The detailed structure of protuberances is shown in the inset of figure 1(e), from which the size of a protuberance was measured to be $350 \pm 42 \mathrm{~nm}$. This nanostructure can be considered as the grating on the compound eye and would possibly enhance the light absorption of the eyes. The protuberance structure and the possible light transmission diagram are schematically shown in figure 1(f). The packed protuberances with typical length $(l)$ of $350 \pm 42 \mathrm{~nm}$ and height $(h)$ of $\sim 120 \mathrm{~nm}$ is within the size range for UV-visible lights.

Biological structures are the result of hundreds of thousands of years of evolution. Such designs of nature possess many unique merits that would be difficult to achieve by a complete artificial simulation. Utilizing them as biotemplates and converting them to inorganic material could be a highly reproducible and low-cost process for fabricating complex nanostructures with unique functions. According to our previous experience in replicating butterfly wings [7], the ALD technique was used to replicate the fly eyes with alumina $\left(\mathrm{Al}_{2} \mathrm{O}_{3}\right)$, which can be conducted below $100^{\circ} \mathrm{C}$ [10-12]. During the growth, the thickness of the alumina layer was precisely controlled by varying the cycles of deposition.

Annealing at $500{ }^{\circ} \mathrm{C}$ for $16 \mathrm{~h}$ in air atmosphere was applied to remove the original fly compound eye template. Under this temperature, the organic corneas of the fly eyes were burned away with the existence of oxygen. Meanwhile, due to the small thickness amorphous alumina layer, it can gradually crystallize into a more robust polycrystalline structure at this temperature [7]. Owing to the excellent uniformity of the alumina film, both the large-scale arrangement of the compound eyes and the nanometer-scale periodic grating structures were perfectly preserved after this vigorous template removing process. As shown in figures 2(a) and (b), the alumina replicas of the fly's eyes exhibited the same shape, orientation and distribution as their 'parent' eyes. Thus, the replica of compound eye structure should be able to keep the 


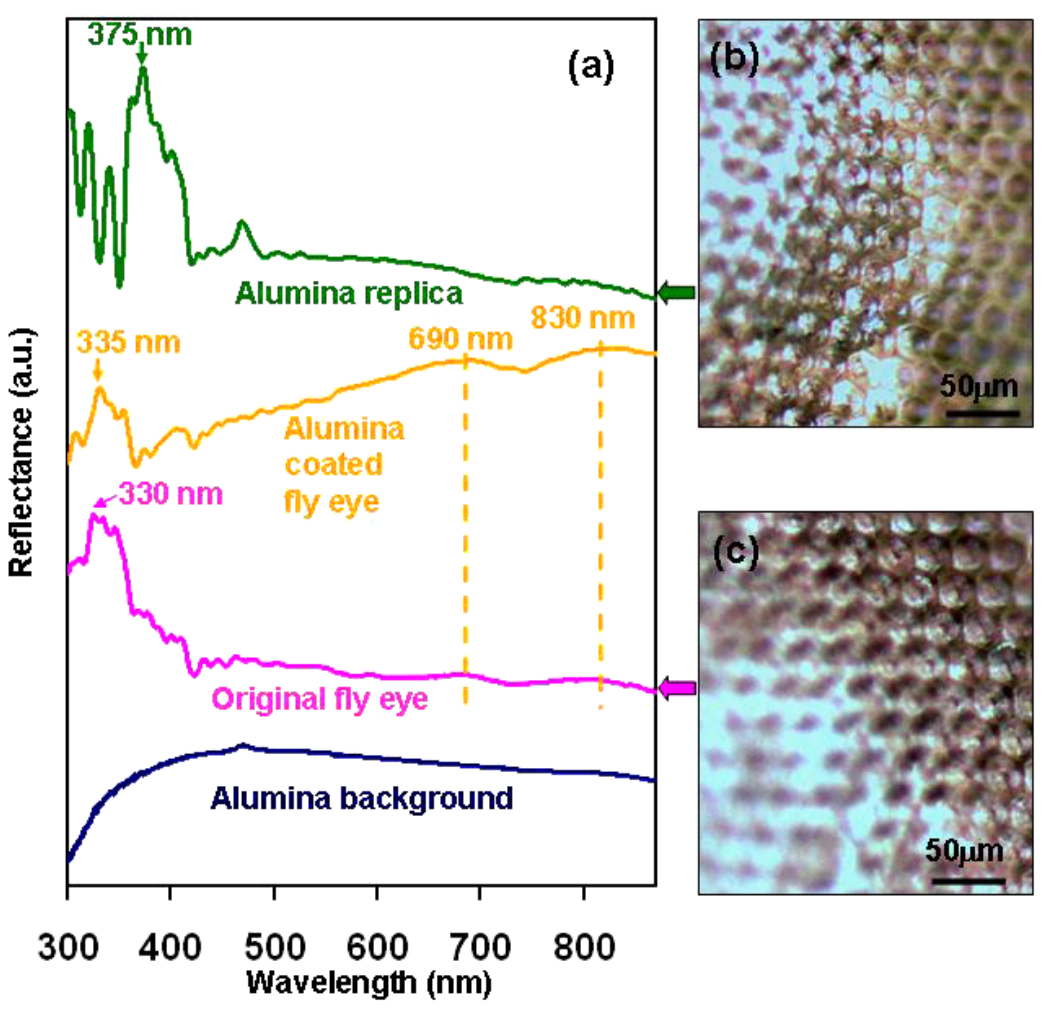

Figure 3. (a) Reflectance spectra recorded on a $100 \mathrm{~nm}$ thick alumina replication of a fly eye, a fly eye coated with $100 \mathrm{~nm}$ alumina thin film, the original fly eye, and a glass substrate coated with $100 \mathrm{~nm}$ alumina thin film as the background signal. The reflectance was measured at $30^{\circ}$ to the normal direction. Optical microscopy images of the alumina replica of fly eyes (b) and the original fly eyes (c).

same wide field of vision. So far, imaging with a field of vision over $90^{\circ}$ was only achieved with fish eye lenses, which relied on bulky and expensive multiple lenses and required stringent alignment. The corresponding energy dispersive $\mathrm{x}$ ray spectrum (EDS) showed that only $\mathrm{O}$ and $\mathrm{Al}$ elements were detected from replicated eyes (figure 2(c), where the $\mathrm{Cu}$ signal was from the TEM grid). The detailed structure of the alumina replica can be clearly observed from a higher magnification SEM image, as shown in figure 2(d). Not only the ommatidium structure, but also the nanosized protuberance on its surface has been precisely replicated by the alumina thin film (figure 2(e)). The corresponding TEM image was recorded from a single piece of an ommatidium, which clearly shows the spherical shape and the linear arrangement of the protuberances (figure 2(f)).

The success of the replication was not only with the morphologies but also with the optical property. Amber diffraction light can be observed on the alumina replica. Angular reflection measurements were performed from the near-UV to visible and near-IR wavelength region to reveal the light interaction with the replicated structures. The reflectance was measured at $30^{\circ}$ to the normal direction, where the angle of incident light was kept at $30^{\circ}$ to the normal direction. The corresponding spectra are summarized in figure 3(a). From top to bottom, the spectra represent the reflectance of a $100 \mathrm{~nm}$ thick alumina replication of a fly eye, a fly eye coated with $100 \mathrm{~nm}$ alumina thin film, the original fly eye, and a glass substrate coated with $100 \mathrm{~nm}$ alumina thin film as the background signal, respectively. The alumina replica exhibited similar optical reflection properties to those of the original fly eye. However, the strongest reflection peak in the near-UV region was red-shifted from 330 to $335 \mathrm{~nm}$ due to the alumina coating induced refractive index change. After the eye template was completely removed, the reflection peak further shifted to $375 \mathrm{~nm}$. The amber color is from the wide reflection peaks from $\sim 600$ to $\sim 700 \mathrm{~nm}$. The corresponding optical microscopy images of the alumina replica and the original fly eyes are shown in figures 3(b) and (c), respectively. The color of alumina replication became a bright amber color compared to the original dark amber color, due to the refraction index change (the organic fly eye material was replaced by inorganic alumina material).

A regular sub-wavelength structure formed on a given surface can significantly reduce the reflection from the surface. Therefore, the protuberances on the fly eyes could be antireflection structures that increase visual efficiency through increased photon capture for a given stimulus. Usually, there are two methods to obtain an antireflection surface. One is to deposit an antireflection coating with media refractive index and exact thickness on the surface of a photonic device by destructive interference. The other is to use a reflective diffraction grating depending on the structure. The simplest diffraction grating is an optical device that has a periodic series of slits in an opaque screen. The compound eye is a complex diffraction grating [13, 14], and the grating would be particularly useful on a curved surface as it would increase the transmission of incident light through the cornea compared to 


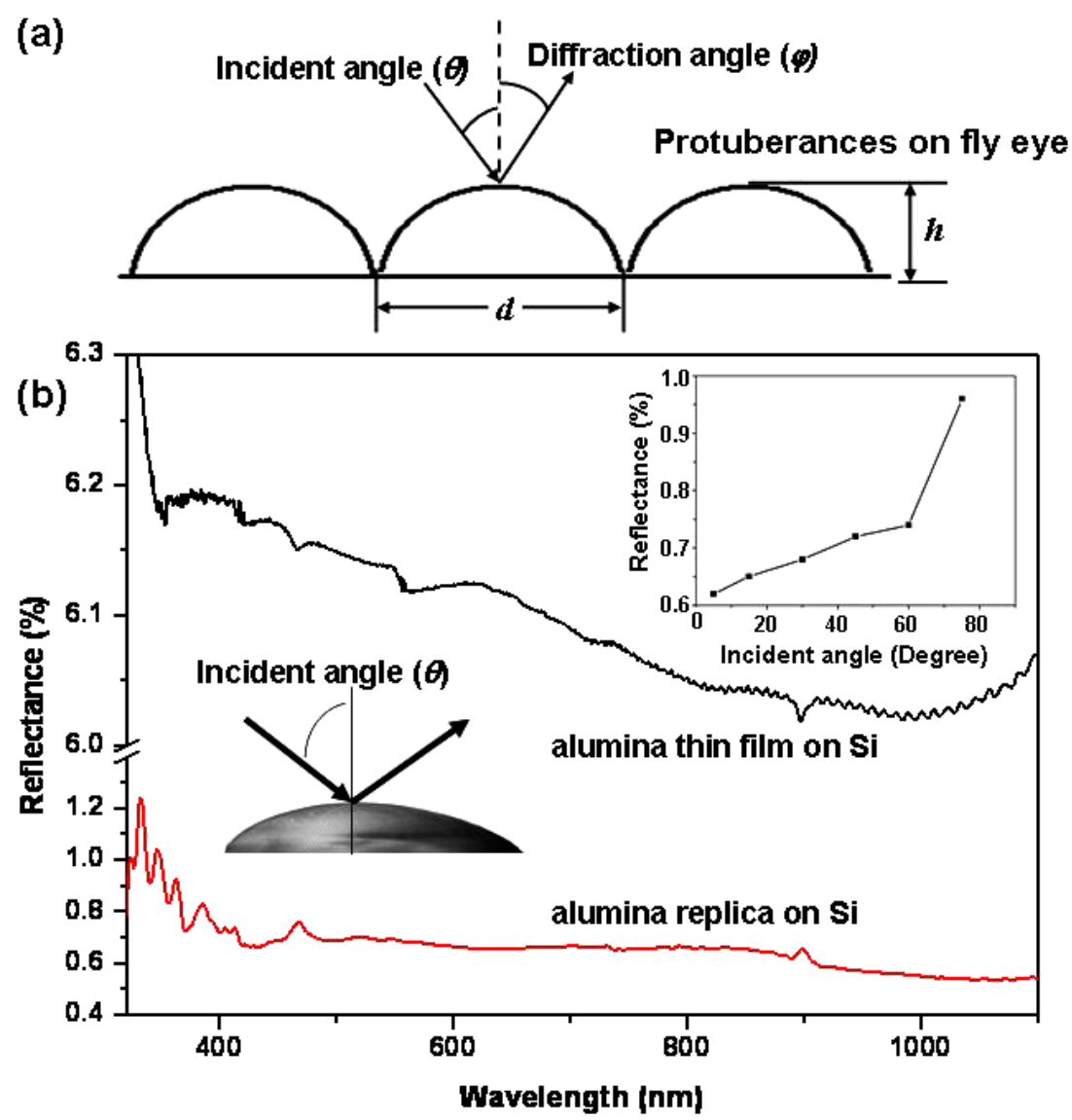

Figure 4. Antireflection property of the alumina replica of the fly eyes. (a) Schematic diagram of the interaction between incident light and protuberance arrays. (b) Reflection spectra of alumina replica of fly eyes (bottom) and alumina thin film with the same thickness (top). Inset: reflectance versus incident light angle, where the data were recorded with a fixed incident light wavelength at $600 \mathrm{~nm}$.

a smooth surface. Such an antireflection grating structure has a potential application in micro optical sensors [15].

For explanation, we simplify the protuberance as triangular gratings, and the antireflection property can be expressed by the grating equation [16]

$$
\sin \varphi_{m}=\sin \theta+m \lambda / d,
$$

where $\theta, \varphi_{m}, m$ and $\lambda$ correspond to incident angle, diffraction angle, diffraction order and wavelength, respectively. For our compound eye grating surface as schematically shown in figure 4(a), $d$ was measured to be $350 \pm 42 \mathrm{~nm}$. In the perfect blazing condition, where $m=-1$, and $\theta$ and $\varphi_{-1}$ were kept at $30^{\circ}$ and $-30^{\circ}$, respectively, the constructive diffraction wavelength is $\lambda \approx 350 \pm 42(\mathrm{~nm})$. This explains the reflection peak existing in the UV band of about $330-375 \mathrm{~nm}$.

In order to investigate the enhanced antireflection property of the replicated fly compound eye structure, angular reflectance was measured on a $100 \mathrm{~nm}$ thick alumina replica and an alumina thin film on silicon substrate with the same thickness. The corresponding spectra are shown in figure 4(b). Within the visible light wavelength range, the alumina film gave a reflectance of $\sim 6.1 \%$, while the fly eye replica only exhibited a reflectance of $\sim 0.7 \%$, which was nearly one order of magnitude lower than that of the thin film with the same thickness. Moreover, the incident light angle showed little fluence on the reflectance. As shown in the inset of figure 4(b), the reflectance of the fly eye replica increased slightly from $0.62 \%$ to $0.73 \%$ when the incident angle increased from $5^{\circ}$ to $60^{\circ}$ accordingly, and reached $0.96 \%$ at $80^{\circ}$. Since the reflectance of the referencing alumina thin film gave a similar increasing trend as the reflectance measured on the fly eye replica, we can conclude that the alumina replica of the fly eye was an efficient antireflection structure of visible light at an incident angle up to $80^{\circ}$.

\section{Conclusion}

In this paper, the fine structure of the household fly compound eye was carefully examined and the entire configuration was completely replicated by alumina through a low-temperature ALD process. The same structure was achieved by removing the fly compound eye template at high temperature and the alumina coating was crystallized simultaneously. The surface of the fly eye is covered by highly packed protuberances, which potentially increases visual efficiency through increased photon capture for a given stimulus. This unique antireflection property was also inherited by the alumina replica. By measuring the reflective spectra of the replica, we demonstrated that the alumina replica of a fly eye was an efficient antireflection structure of visible light at an 
incident angle up to $80^{\circ}$. Such a grating would be particularly useful on a cured corneal surface since it could increase the transmission of incident light through the cornea compared with a smooth surface. The fly eye replica with antireflection structure exhibits great potential in the applications of optical coating, sensing or lens arrays.

\section{Acknowledgments}

This research was supported by the National Science Foundation (NSF) and Centers of Cancer Nanotechnology Excellence (CCNE) from National Institute of Health (NIH). Dr Huang is grateful for the support of the Chinese study abroad fund.

\section{References}

[1] Davis S A, Burkett S L, Mendelson N H and Mann S 1997 Nature 385 420-3

[2] Cook G, Timms P L and Spickermann C G 2003 Angew. Chem. Int. Edn 42 557-9
[3] Seshadri R and Meldrum F 2000 Adv. Mater. 12 1149-51

[4] Chia S, Urano J, Tamanoi F, Dunn B and Zink J I 2000 J. Am. Chem. Soc. 122 6488-9

[5] Yang D, Qi L M and Ma J M 2002 Adv. Mater. 14 1543-6

[6] Dong A, Wang Y J, Tang Y, Ren N, Zhang Y, Yue Y and Gao Z 2002 Adv. Mater. 14 926-9

[7] Huang J Y, Wang X D and Wang Z L 2006 Nano Lett. $62325-31$

[8] Stavenga D G 2002 J. Comp. Physiol. A 188 337-48

[9] Stavenga D G 2002 J. Exp. Biol. 205 1077-85

[10] Groner M D, Fabreguette F H, Elam J W and George S M 2004 Chem. Mater. 16 639-45

[11] Ott A W, Klaus J W, Johnson J M and George S M 1997 Thin Solid Films 292 135-44

[12] Langereis E, Creatore M, Heil S B S, van de Sanden M C M and Kessels W M M 2006 Appl. Phys. Lett. 89081915

[13] Parker A R, Hegedus Z and Watts R A 1998 Proc. R. Soc. B 265 811-5

[14] Miller W H, Moller A R and Bernhard C G 1966 The corneal nipple array The Functional Organization of the Compound Eye ed C G Berngard (Oxford: Pergamon) pp 21-33

[15] Lee C, Bae S Y, Mabasser S and Manohara H 2005 Nano Lett. $52438-42$

[16] Jull E V, Beaulieu N C and Hui D C W 1984 J. Opt. Soc. Am. A 1 180-2 